\title{
Trajectory Planning for a Human-Robot Interaction Rehabilitation System using Direct-Collocation Optimization
}

\author{
Arash Hashemi, John McPhee \\ University of Waterloo \\ 200 University Ave W., Waterloo, Canada \\ a8hashem@uwaterloo.ca; mcphee@uwaterloo.ca
}

\section{Extended Abstract}

One of the essential considerations in Human-Robot Interaction (HRI) research is the trajectory planning phase [1]. This issue becomes vital in rehabilitation robotics due to the sensitive nature of the problem and patient involvement. An appropriate trajectory is conducive to improved clinical progress whereas an unsuitable practice can lead to the aggravation of the patient status. Due to the prevalence of stroke in recent years, this study aims to find the task-space manipulation trajectory for a 2 degrees of freedom (DOF) post-stroke end-effector-based rehabilitation robot, developed by Quanser, the Toronto Rehabilitation Institute (TRI), and the University of Waterloo.

This planar robot provides upper-limb repetitive practices by moving the patient's hand in the horizontal plane. The model of the robot is coupled with a 2D upper extremity musculoskeletal model, consisting of 6 lumped muscles, to form the HRI nonlinear dynamic system [2,3]. This model includes four states, 2 robot angles and angular velocities, and 8 inputs, 2 robot motor torques and 6 arm muscle activations. An optimization-based trajectory planning scheme is presented and a multi-variable single-phase continuous-time optimization problem is set up to acquire the end-effector trajectory. Previous research on the trajectory planning of rehabilitation robots has considered a predefined path and found the corresponding parameters $[4,5]$. In this study, however, the decision variables are free at the optimization points and splines are used only after optimization, in the interpolation phase. Other studies have considered kinematic and skeletal dynamic human models in the planar frameworks without considering muscles [6,7]. The integration of the human arm model with muscle dynamics in the optimization has the merit of producing a clinically plausible solution.

A direct-collocation method is used to solve the problem, with the help of an open-source optimization library in MATLAB called "OptimTraj" [8]. The decision variables are the robot states, and the HRI nonlinear dynamics is considered as the equality constraint:

$$
\dot{x}=f(x, u, t)
$$

The problem also includes inequality box constraints on both the states and the inputs:

$$
\begin{array}{ll}
x_{i \min } \leq x_{i} \leq x_{i \max } & \text { for } i=1: N_{s} \\
u_{j \min } \leq u_{j} \leq u_{j \max } & \text { for } j=1: N_{u}
\end{array}
$$

where $N_{s}$ is the number of states, and $N_{u}$ is the number of inputs. The initial and final states, and time, are fixed. This boundary constraint results in a point-to-point reaching planar trajectory, which is common in rehabilitation practices. Fixing the final time will set the angular velocity of the robot joints, which determines the rehabilitation pace. Trapezoidal transcription is used to discretize the dynamics and constraints. The output of the optimization is the joint trajectory of the robot and the open-loop robot and arm inputs.

A composite cost including robot input and input rate, arm muscle activations, and arm joint accelerations resulted in the best manipulation trajectory [9]. The planner provides the open-loop control inputs to follow the desired trajectory. However, a state feedback controller is required to stabilize the trajectory. To this end, a Linear Quadratic Controller 
(LQR) is applied on the robot. The results show trajectory tracking with good accuracy. Future work will include applying other controllers for following this trajectory.

\section{References}

[1] Q. Meng, Q. Xie, Z. Deng, and H. Yu, "A general kinematics model for trajectory planning of upper limb exoskeleton robots," International Conference on Intelligent Robotics and Applications, 2019, vol. 11745 LNAI, pp. 63-75.

[2] B. Ghannadi, N. Mehrabi, and J. McPhee, "Development of a human-robot dynamic model to support model-based control design of an upper limb rehabilitation robot,". In Barcelona: ECCOMAS Thematic Conference on Multibody Dynamics, 2015.

[3] N. Mehrabi, R. S. Razavian, and J. McPhee, "A Physics-Based Musculoskeletal Driver Model to Study Steering Tasks," J. Comput. Nonlinear Dyn., vol. 10, no. 2, pp. 1-8, 2015.

[4] G. Rosati, G. Volpe, and A. Biondi, "Trajectory planning of a two-link rehabilitation robot arm," IFToMM World Congress, 2007.

[5] H. Kalani, A. Akbarzadeh, S. N. Nabavi, and S. Moghimi, "Dynamic modeling and CPG-based trajectory generation for a masticatory rehab robot," Intell. Serv. Robot., vol. 11, no. 2, pp. 187-205, 2018.

[6] Sh. Tang, L. Chen, M. Barsotti, L. Hu, Y. Li, X. Wu, L. Bai, A. Frisoli, and W. Hou. "Kinematic synergy of multiDOF movement in upper limb and its application for rehabilitation exoskeleton motionplanning,". Frontiers in Neurorobotics, 13(November), 2019.

[7] A. J. Nagengast, D. A. Braun, and D. M. Wolpert. "Optimal control predicts human performance on objects with internal degrees of freedom,". PLoS Comput Biol,5(6):e1000419, 2009

[8] M. Kelly, "An introduction to trajectory optimization: How to do your own direct collocation,". SIAM Rev., vol. 59, no. 4, pp. 849-904, 2017.

[9] B. Berret, E. Chiovetto, F. Nori, and T. Pozzo, "Evidence for composite cost functions in arm movement planning: an inverse optimal control approach,". PLoS Comput Biol, 7(10), e1002183, 2011. 